\title{
Uma Ferramenta para Recomendação Pedagógica Baseada em Mineração de Dados Educacionais
}

\author{
Ranilson Paiva' ${ }^{1}$ Ig Ibert Bittencourt ${ }^{1}$, Alan Pedro da Silva ${ }^{1}$ \\ ${ }^{1}$ Instituto de Computação - Universidade Federal de Alagoas (UFAL) \\ Av. Lourival Melo Mota, s/n - Tabuleiro do Martins, CEP 57.072-970 Maceió, AL - Brazil \\ \{roap,ig.ibert, alapedro\} @ic.ufal.br
}

\begin{abstract}
Resumo. A dissertação trata da conceitualização e criação de uma ferramenta para a recomendação pedagógica cujo objetivo é prover aos professores de cursos baseados na web, recomendações pedagógicas personalizadas geradas por especialistas no domínio com base nos resultados da mineração dos dados educacionais dos alunos. A ferramenta segue o Processo de Recomendação Pedagógica e foi utilizada em um estudo de caso com dados reais de um curso de língua Espanhola com 200 alunos. Os resultados permitiram detectar padrões de interação úteis, usados na criação de recomendações, avaliadas (relevância) por especialistas no domínio educacional/pedagógico, e disponibilizadas para que os professores as oferecessem a seus alunos.
\end{abstract}

\section{INTRODUÇÃO}

A motivação que levou à escrita desta dissertação está relacionada com os índices que medem a qualidade do ensino e da educação no Brasil, nos âmbitos nacional e internacional. Conhecer tais índices nos levou a uma busca pelos desafios, planejamentos, objetivos e metas governamentais, dentro do contexto da informática na educação, para que pudéssemos vislumbrar uma forma de contribuir significativamente com os esforços que visam sanar esse problema.

Primeiramente observou-se o PISA (Programa para a Avaliação Internacional dos Alunos), que avalia os alunos concluindo a escolaridade básica obrigatória em diversos países, para avaliar o quão bem essas escolas estão preparando esses jovens para exercer a cidadania. O Brasil ficou em $\mathbf{5 8}^{\circ}$ lugar, de um total de 75 países avaliados [WALKER, 2011]. Em seguida analisou-se o IDEB (Índice de Desenvolvimento da Educação Básica) que mede a qualidade do ensinos fundamental e médio das redes de ensino do país. Em 2011, as médias, para um total de 10.0 pontos, os resultados foram: 5.0 para os anos iniciais do ensino fundamental, 4.1 para os anos finais do ensino fundamental e 3.7 para o ensino médio [MEC; IDEB, 2012], constatando que não é boa a situação da educação no Brasil. De posse desses dados e buscou-se as demandas para a educação no Brasil, expressas por meio do Plano Nacional da Educação, documento onde são definidos os objetivos e orientações para a educação nacional em um período de 10 anos [PNE, 2010].

Já percebendo esse problema com a educação, e outros desafios, a Sociedade Brasileira de Computação (SBC) publicou o documento intitulado "Grandes Desafios da Computação 2006 - 2016" [DE CARVALHO, 2006], criado com o objetivo de motivar e guiar as pesquisas em computação no país. Dos cinco desafios mencionados, um deles se refere ao "Acesso Participativo e Universal do Cidadão Brasileiro ao Conhecimento". 
Para reforçar seu comprometimento com esses problemas, a SBC criou o Workshop de Desafios da Computação Aplicada à Educação (DesafIE!), com o objetivo de debater temas relacionados aos desafios em educação, sob a perspectiva computacional [DesafIE!, 2012], como: a adaptação do conteúdo ao aprendiz, o registro de longo prazo dos perfis de aprendizes e tutores, a mineração de dados educacionais em busca de padrões de aprendizagem e a personalização da aprendizagem.

Autores relatam crescimento do interesse e do acesso à educação, contudo acompanhado de necessidades que direcionam a educação para um novo paradigma de aprendizagem, mediado pela tecnologia e possibilitando o aprendizado a partir de qualquer lugar, a qualquer momento e para qualquer pessoa, AAAL [BITTENCOURT, 2008; 2009]. A adoção desse paradigma aumentou a oferta de cursos online oferecidos por intituições renomadas nacionais e internacionais, que utilizam ambientes de aprendizagem como: Moodle, Teleduc, Blackboard, Sakai e, mais recentemente, os MOOCs como EDX, Coursera e Udacity cujo objetivo é prover interatividade em larga escala (razão de serem denominados "massivos"), através de recursos educacionais como vídeos, exercícios, fóruns, avaliações por pares, dentre outros.

Contudo, ao se analisar os dados do progresso de um curso em uma plataforma MOOC [DUKE, 2013], nota-se que alguns dos problemas identificados (grande número de desistentes, alunos com problemas de desempenho) não foram satisfatoriamente soluciondos. Tais problemas surgem em decorrência da considerável quantidade e diversidade de dados gerados a partir das interações dos usuários com esses ambientes.

Mesmo que o ambiente ofereça alguma ferramenta para identificar e apresentar os problemas de cada um dos alunos, o professor não conseguirá, em tempo hábil, analisar essas informações e tomar uma decisão que solucione essas necessidades. Com base nisso surgiram questões de pesquisa: (Q1) Como processar esses dados de modo a extrair deles informações úteis e relevantes? (Q2) $\underline{O}$ que fazer com o resultado obtido a partir do processamento desses dados educacionais? (Q3) Como tornar o processo transparente para os professores?

Identificou-se, então, a necessidade de um suporte computacional capaz de auxiliar o professor no processo de tomada de decisões pedagógicas. Isto pode ser feito utilizando técnicas de mineração de dados educacionais (Q1) cujo objetivo é o de aprimorar os processos de ensino e aprendizagem, com base nos dados provenientes de ambientes educacionais [ROMERO, 2011]. Utilizando, então, os resultado dessa mineração para propor recomendações pedagógicas (Q2), as quais alimentarão um sistema de recomendação que sugerirá itens (recursos educacionais) a serem consumidos pelos usuários (os alunos) de acordo com suas necessidades e características [RICCI, 2011]. Por fim isso deve ser oferecido por meio de uma ferramenta capaz de abstrair a complexidade das técnicas utilizadas, não necessitando dos professores conhecimento especializados sobre as mesmas o que, por fim, responde à nossa (Q3).

\section{O PROCESSO DE RECOMENDAÇÃO PEDAGÓGICA}

A proposta apresentada é a criação de uma Ferramenta para Recomendação Pedagógica, que disponibiliza aos professores de cursos baseados na web, recomendações pedagógicas personalizadas por meio da mineração dos dados 
educacionais oriundos das interações dos alunos com o ambiente de aprendizagem. Para alcançar esse objetivo criou-se um processo para coordenar a geração dessas recomendações, cujas etapas são exibidas na figura 1 e detalhadas nessa seção.

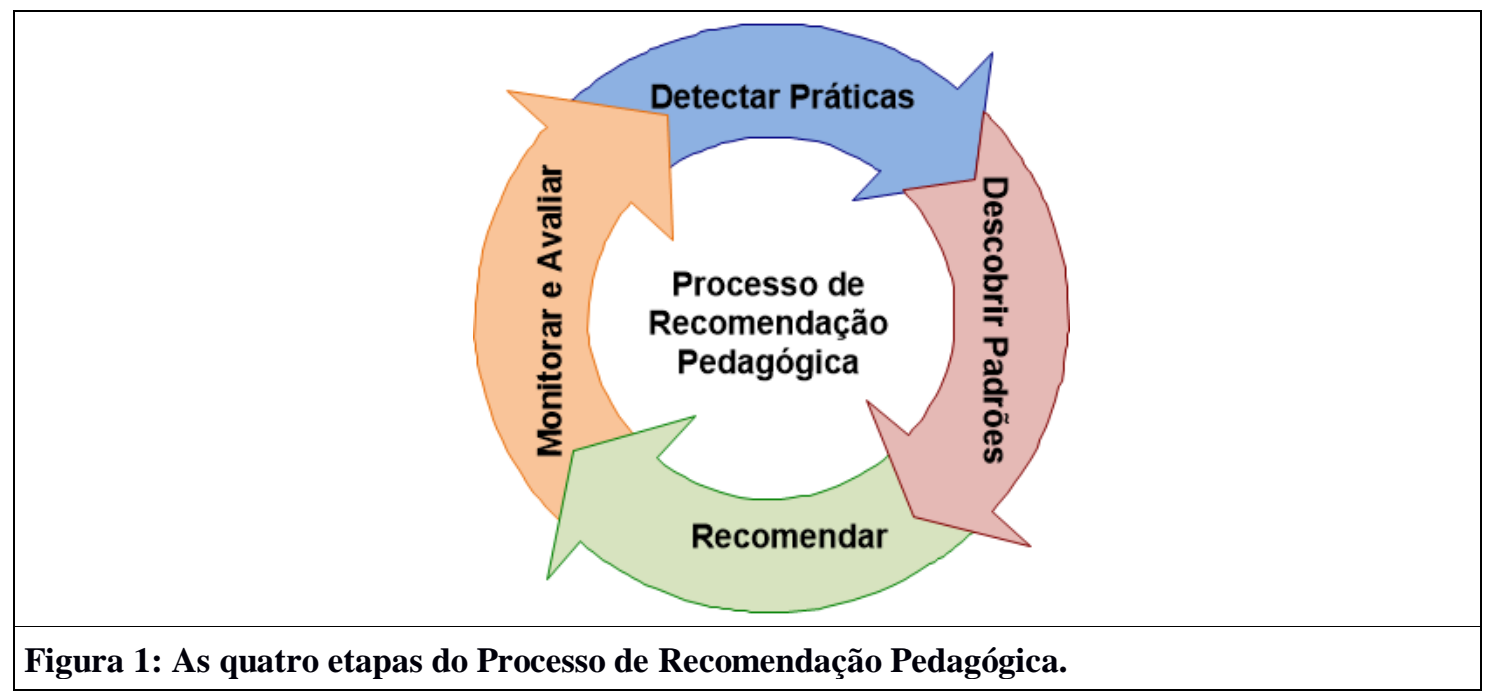

O objetivo da detecção de práticas é identificar se as interações dos alunos, dentro do ambiente de aprendizagem, estão resultando em progresso na aprendizagem, individual ou em grupo, ou se os resultados observados indicam o contrário. Não é objetivo dessa etapa identificar os detalhes e padrões que explicam essas práticas. São exemplos de práticas a identificação de resultados de provas abaixo da média, a detecção de alunos com número de faltas, para um determs0inado período, superior à média ou a um valor de referência, etc.

$\mathrm{Na}$ descoberta de padrões analisa-se os dados interacionais para descobrimos os padrões relacionados às práticas, as quais foram detectadas na primeira etapa, ou seja, descobrir o motivo pelo qual cada prática está ocorrendo. Isso ocorre através da avaliação de hipóteses (possíveis explicações para as práticas) utilizando técnicas de mineração e análise estatística dos dados com o intuito de confirmar, ou rejeitar, essas hipóteses.

$\mathrm{Na}$ etapa seguinte, recomendar, as recomendações pedagógica serão disponibilizadas para os professores, os quais encaminharão as mais apropriadas para determinado aluno, dada a situação pedagógica do mesmo. Essas recomendações são criadas por especialistas no domínio pedagógico com base na análise dos padrões descobertos na etapa anterior, e têm como foco solucionar situações pedagógicas trazidas à luz por meio dos padrões descobertos. As Recomendações Pedagógicas são entidades externas ou próprias do ambiente de aprendizagem, cujo objetivo é oferecer orientações para superar dificuldades pedagógicas já detectadas (i.e.: latentes), ou previstas (i.e.: em potencial). Uma recomendação pedagógica útil é aquela capaz de aprimorar a experiência de aprendizagem dos alunos para os quais ela foi direcionada.

Para que se possa recomendar algo através de um ambiente de aprendizagem, precisamos conhecer quais recursos educacionais estão disponíveis. Esses recursos localizam-se na interface dos usuários entre si (i.e.: professores, tutores e alunos), e entre os usuários com o ambiente de aprendizagem, tendo como finalidades apresentar o conteúdo (aulas em diversos formatos de mídia), avaliar a aquisição de conhecimento 
(exercícios e testes), coletar e apresentar resultados e dados educacionais (gráficos e relatórios), prover ajuda e apoio (tutoriais, material extra, links externos), comunicar, engajar e agregar seus usuários (chat e fórum).

$\mathrm{Na}$ etapa de monitoramento e avaliação o desempenho dos alunos é avaliado quanto ao progresso em decorrência das recomendações recebidas e, também, se avalia o quão relevantes estão sendo essas recomendações para as situações pedagógicas para as quais elas foram propostas. Essa tarefa é realizada da seguinte forma:

\section{OS CENÁRIOS DE MINERAÇÃO}

Um dos requisitos da ferramenta foi o de não demandar dos professores, conhecimento avançado sobre as técnicas utilizadas. Por esse motivo foi importante a criação de uma forma de encapsular os requisitos de mineração de dados, associando-os aos objetivos pedagógicos. Isso foi denominado de Cenário de Mineração.

Para definir cada cenário foi preciso determinar (1) Qual o objetivo do cenário? (2) Quais os dados necessários para atingir esse objetivo? e (3) Como esses dados devem ser processados para se atingir os objetivos? Os cenários criados abordam questões de interesse pedagógico como a avaliação da qualidade das interações dos alunos no ambiente de aprendizagem [MORAN, 2006], a previsão de resultados de provas e avaliações [PARK, 2007, 2009] e o agrupamento os alunos por nível de participação e engajamento com o curso [BAYER, 2012]. Para responder à terceira pergunta foi consultado [ROMERO, 2011] e listou-se as possiveis tarefas de mineração de dados para cada cenário. Os resultados estão sumarizados na tabela 1.

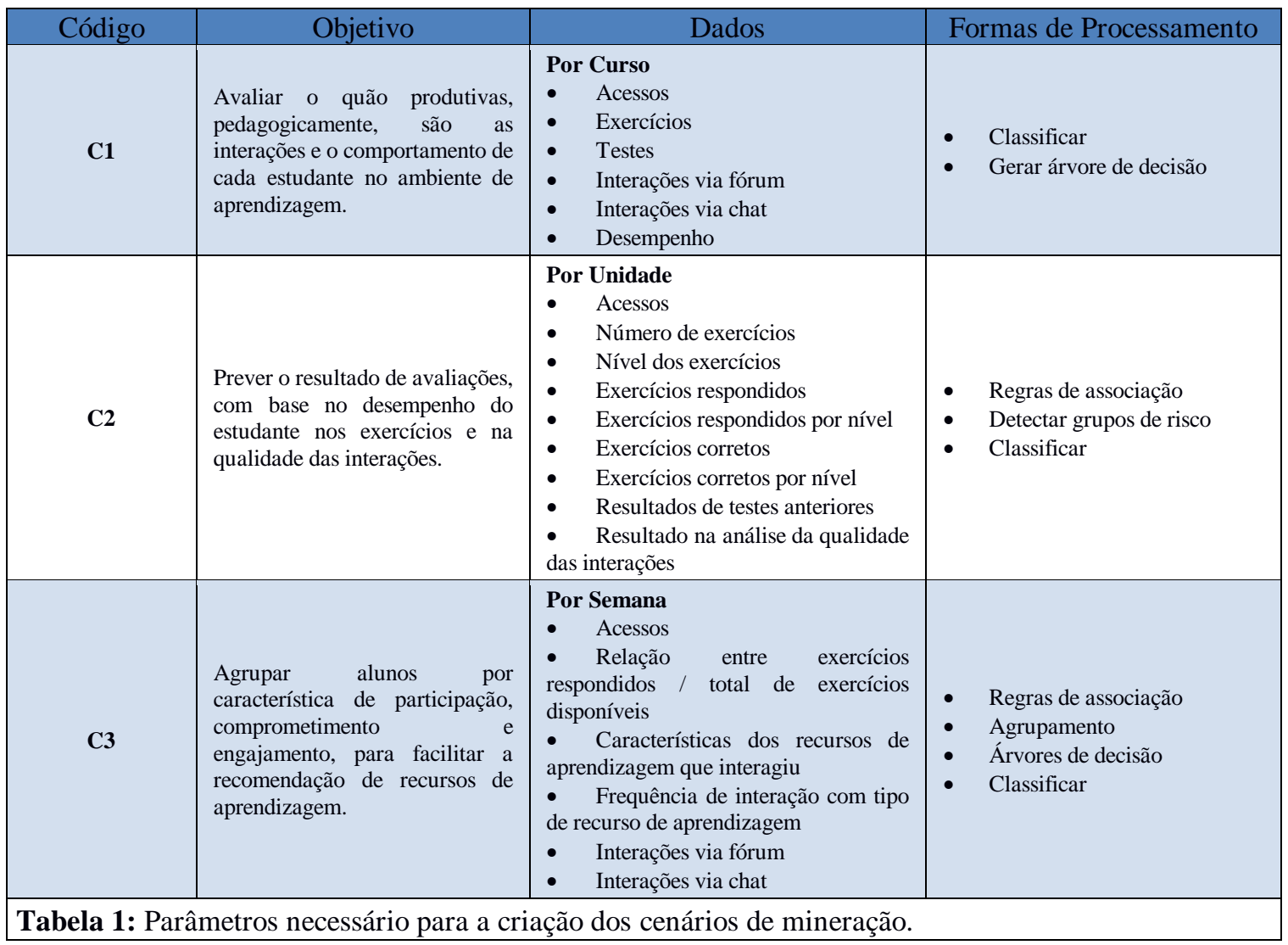


Posteriormente, para que as definições exibidas na tabela 1 tornassem-se, de fato, um cenário de mineração foi preciso: escolher qual a tarefa de mineração mais apropriada para o objetivo especificado em cada cenário, avaliar a disponibilidade de dados no sistema utilizado [UFALLínguas, 2012] e, também, determinar com detalhes como se daria o processo de mineração dos dados (o pré-processamento, a mineração e o pós-processamento dos dados e dos resultados).

O pré-processamento, de forma geral, consistiu de remoção de valores maiores e menores que o triplo da variação interquartil ( $\mathrm{IQR}^{1}$ ), classificados como outliers. Também foram removidos os registros dos alunos desistentes e o campo de identificação (ID), pois para os algoritmos escolhidos esses valores influenciam nos resultados. Além disso, por recomendação quanto ao desempenho, os valores não numéricos e categóricos foram transformados em valores numéricos de ponto flutuante. Por fim os dados foram dispostos em um arquivo no formato ARFF, que é aceito nativamente pela API utilizada no experimento (WEKA® API).

Para a mineração dos dados selecionamos as técnicas de Árvore de Decisão (J48), Árvores de Classificação e Regressão (SimpleCart), Agrupamento (Simple KMeans), assoiando o objetivo do cenário com as descrições dos algoritmos, segundo [WU, 2007; WITTEN, 2011; HAN, 2011], atentando, também, para a correta escolha dos parâmetros [BOUCKAERT, 2012].

Por fim, no pós-processamento dos dados, seguimos as orientações de [GIBERT, 2008] oferecendo uma forma possível de entender os resultados, comunicalos para uma etapa seguinte do processo ou encaminhá-los diretamente àqueles responsáveis pela tomada de decisão, de forma que permitissem identificar informações relevantes com rapidez e de maneira simples para o usuário final. Desfizemos as transformações realizadas nos dados originais, recolocamos os identificadores dos registros e geramos visualizações para apresentar os resultados. A descrição de cada cenário encontra-se na tabela 2.

\section{A FERRAMENTA PARA A RECOMENDAÇÃO PEDAGÓGICA}

A ferramenta foi criada em Java ${ }^{2}{ }^{2}$ reutilizando a implementação dos algoritmos de mineração escolhidos por meior da API Weka®. A capacidade de reuso de código, escalabilidade e a independência de algumas partes que constituem a ferramenta foram definidas como requisitos do projeto, por tal motivo a ferramenta foi desenvolvida seguindo os princípios do Processo de Desenvolvimento Baseado em Componentes [SOMMERVILLE, 2011] por meio do modelo COSMOS $^{3}$ [GAYARD, 2008].

A ferramenta está divida em dois módulos: Módulo de Recomendação Pedagógica, através da qual os professores podem interagir com as recomendações pedagógicas e encaminhá-las para os alunos, ou turmas, apropriados bem como avaliar e monitorar o impacto das recomendações no desempenho acadêmico deles. Essas recomendações, por sua vez, que são geradas por especialistas no domínio educacional

\footnotetext{
${ }^{1}$ Interquartile Range, em Inglês.

${ }^{2}$ Disponível em: http://www.java.com/en/

3 Em Inglês: COmponent System MOdel for Software architectures (Modelo de Sistema de Componentes para Arquiteturas de Software).
} 
com auxílio dos resultados do Módulo de Mineração dos Dados Educacionais, cujas tarefas são orientadas pelos Cenários de Mineração, detalhados na seção 3.

\begin{tabular}{|c|c|c|c|}
\hline 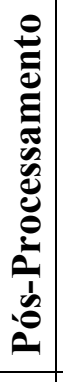 & 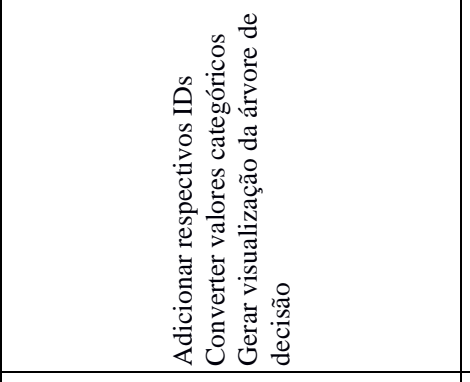 & 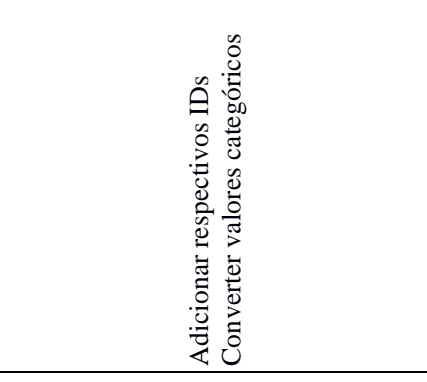 & 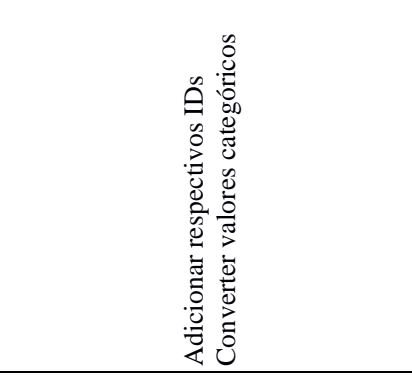 \\
\hline 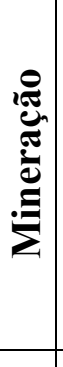 & 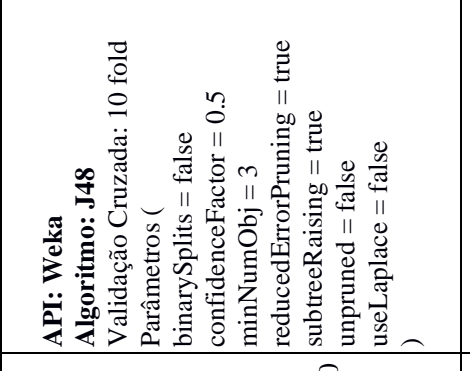 & 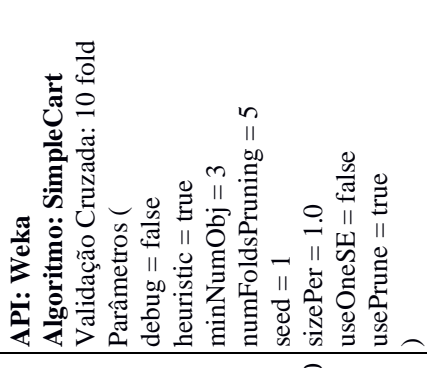 & 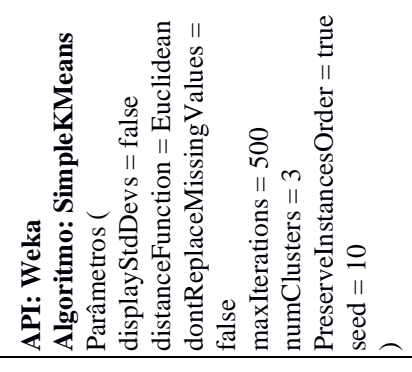 \\
\hline 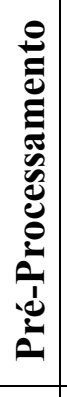 & 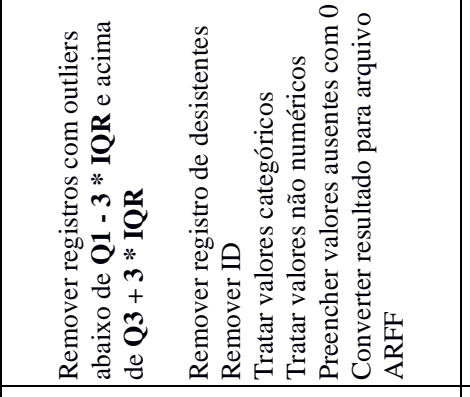 & 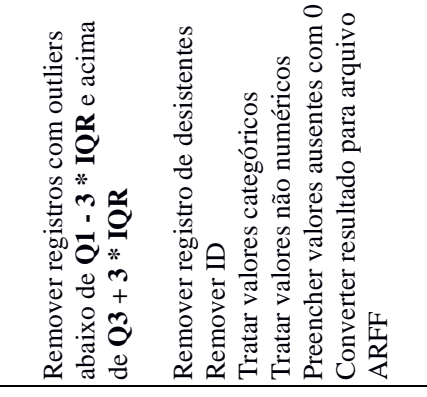 & 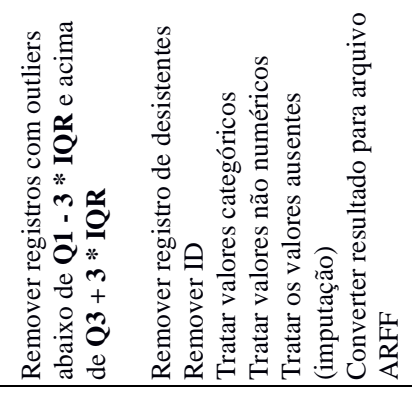 \\
\hline 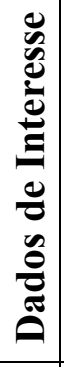 & 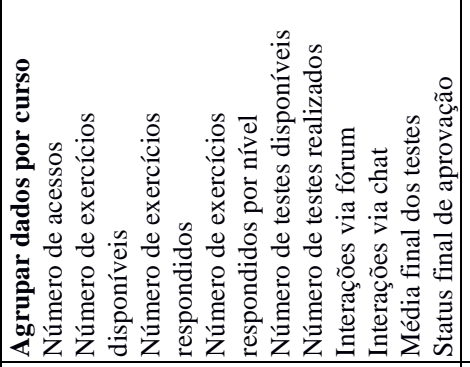 & 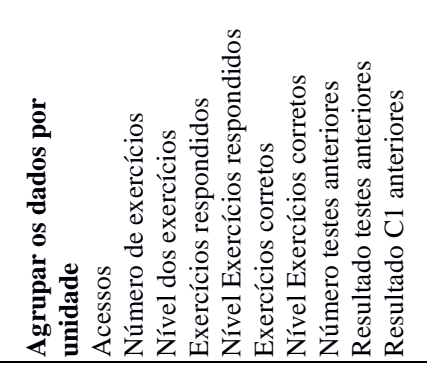 & 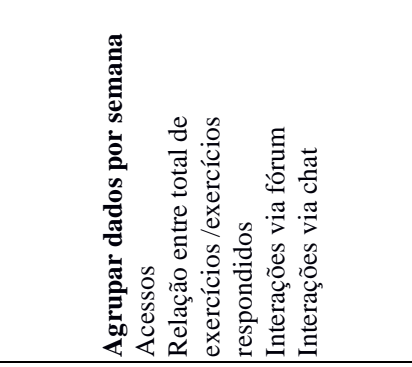 \\
\hline & $\bar{U}$ & U & $\tilde{U}$ \\
\hline
\end{tabular}

Para ter acesso às recomendações pedagógicas personalizadas o professor deve selecionar o cenário de interesse. Com isso o módulo de mineração executará o processo sobre os dados educacionais, em busca de padrões de interesse pedagógico, de acordo com os objetivos e parâmetros definidos no cenário. Os resultados são utilizados para determinar que recomendações são compatíveis com as situações pedagógicas que os alunos, ou turmas, estão vivenciando. Um lista com cinco recomendação (as quatro melhor ranqueadas e uma aleatória), para cada alunos em dificuldade, serão apresentadas ao professor, que deverá escolher a mais apropriada e encaminha-la ao(s) aluno(s). Sendo aprovada, a recomendação escolhida receberá uma pontuação positiva (avaliação da recomendação), em caso contrário as recomendações serão 
desconsideradas (i.e.: o aluno terá uma "necessidade não tratada"), e as recomendações serão pontuadas negativamente (para o cenário em questão).

O desempenho dos alunos expostos às recomendações será monitorado para que se possa avaliar estas proveram melhoria em sua experiência de aprendizagem. Para isso compara-se a taxa de sucesso ${ }^{4}$, dos alunos, nos recursos educacionais prórpios da unidade, com a taxa de sucesso nos recursos educacionais recomendados.

A contribuição dessa ferramenta de recomendação é a de realizar esse processo sem que os professores necessitem de conhecimento sobre as técnicas envolvidas, bastando conhecer os objetivos pedagógicos dos cenários de mineração disponíveis.

Apresentamos na figura 2 a Arquitetura de Implementação, através do diagrama de componentes.

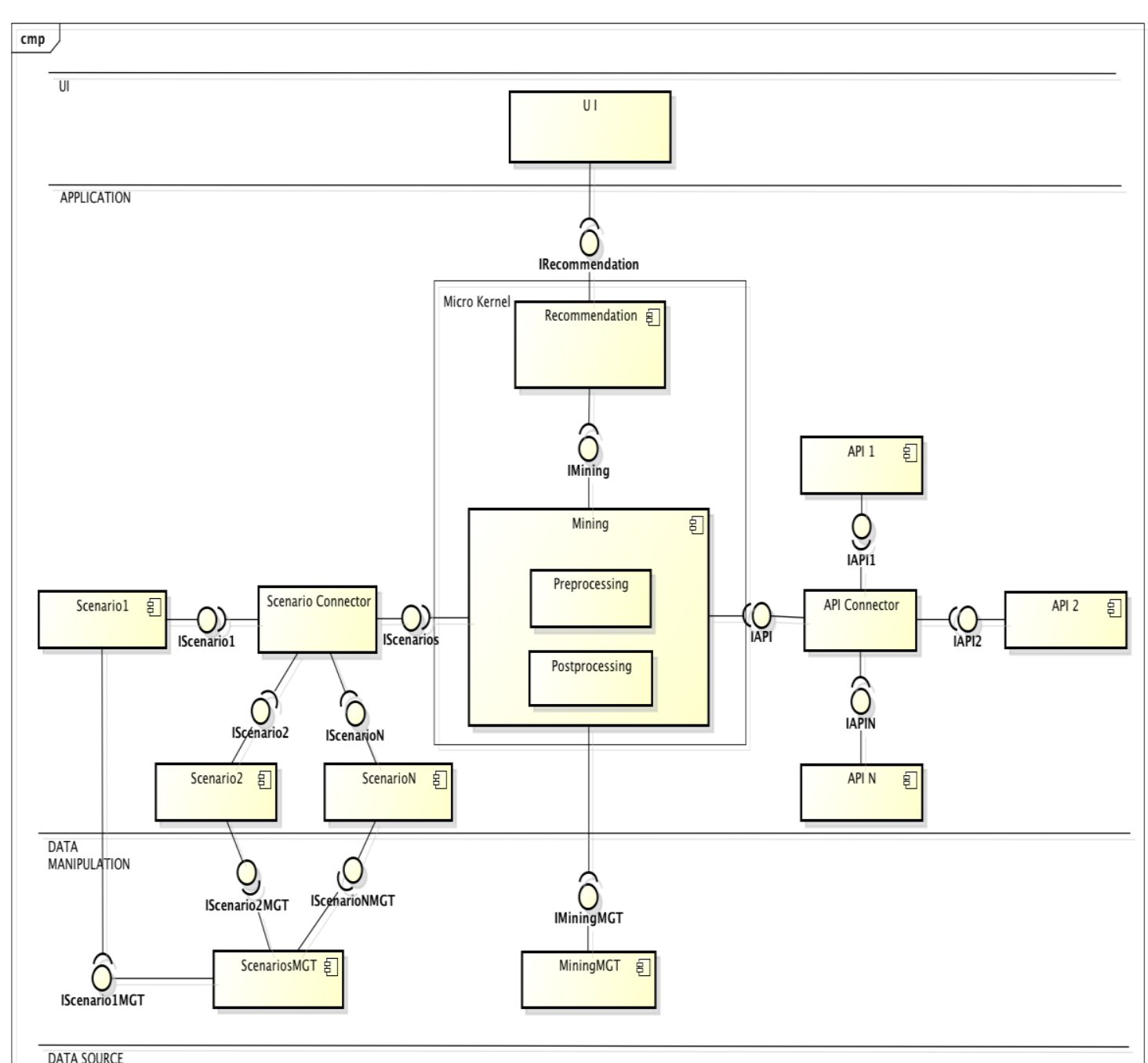

Figura 2: Diagrama de Componentes.

\footnotetext{
${ }^{4}$ A taxa de sucesso refere-se a assistir completamente um vídeo, responder uma atividade extra, realizar uma postagem em um fórum, dentre outras ações com valor pedagógico.
} 


\section{ESTUDO DE CASO}

A ferramenta foi utilizada em um cenário educacional real. O cenário em questão foi um curso de línguas, UFAL Línguas - Espanhol, oferecido na modalidade online, que ocorreu de 22 de Outubro de 2012 a 10 de Fevereiro de 2013, e teve 200 alunos matriculados, os quais geraram mais de 700 megabytes de dados.

Nesse estudo de caso aplicamos o cenário de mineração número 1: avaliação da qualidade das interações dos alunos com o ambiente de aprendizagem, definido na tabela 1. Para isso os dados do curso foram analisados seguindo o processo de recomendação pedagógica, bem como as definições apresentadas na tabela $\mathbf{2}$, resultando na representação visual de uma árvore de decisão (figura 3), que foi utilizada para inferir recomendações associado-as às situações pedagógicas identificadas. No final desse processo, com o propósito de lidar o problema de início frio ${ }^{5}$, uma pesquisa de opinião foi realizada (com professores de línguas de outras instituições) para determinar a relevância dessas recomendações para o cenário de mineração abordado. O resultado pode ser visto na tabela 3 .

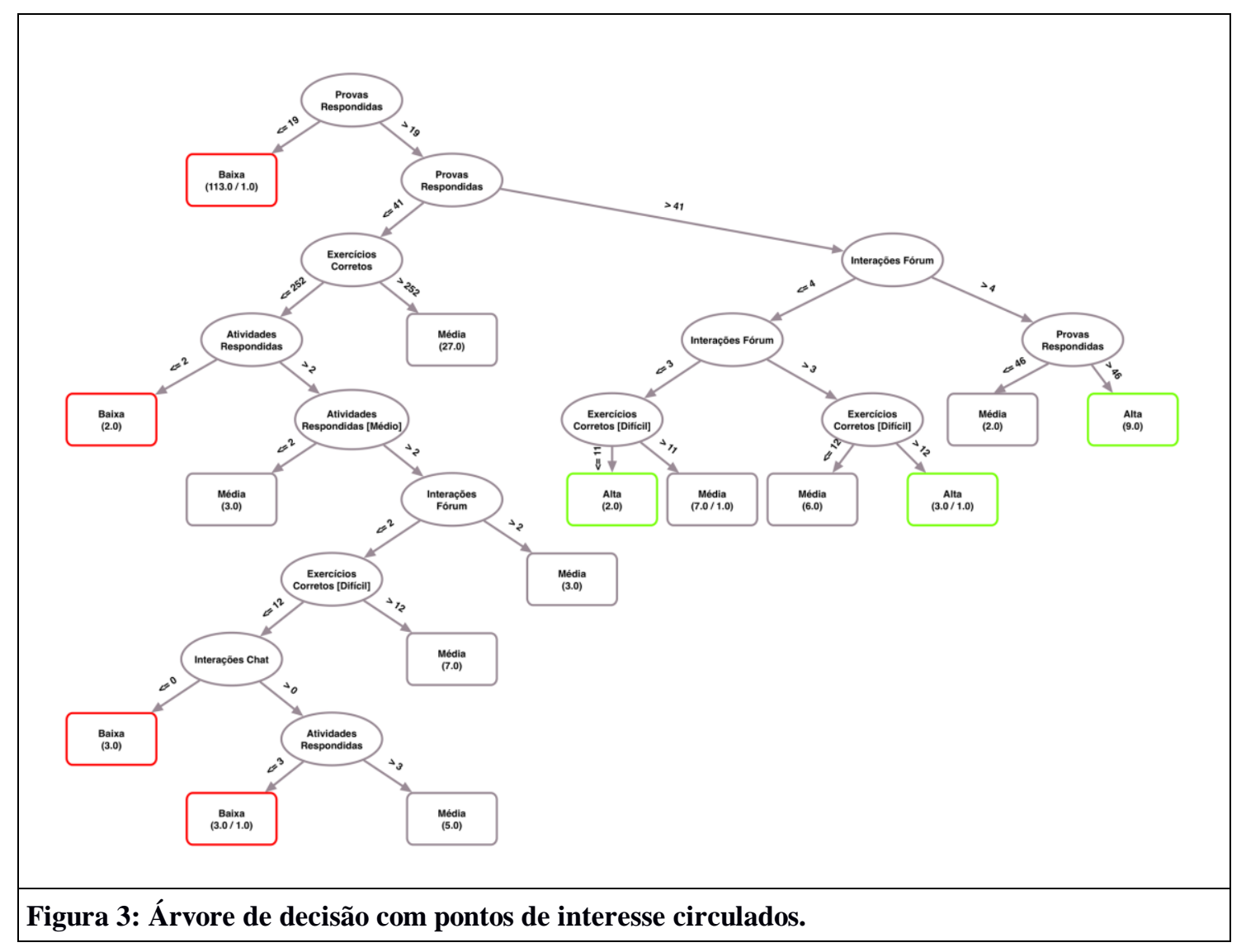

\footnotetext{
${ }^{5}$ Cold start: problema de recomendação de ocorre quando um item de recomendação não possui pontuação que o faça aparecer em um resultado.
} 


\begin{tabular}{|c|c|c|c|c|c|c|c|c|c|c|c|c|c|c|c|}
\hline Regra de Associação & Recomendação & \multicolumn{14}{|c|}{ Relevância } \\
\hline \multirow{5}{*}{ IF provasRespondidas $<=19$} & ProvasNaoRespondidasPenalidade[0] & 5 & & & & 5 & & & & & & & & & \\
\hline & $\begin{array}{l}\text { ProvasNaoRespondidasPenalidade[50] } \\
\end{array}$ & 1 & & & & 1 & & 5 & & 4 & & & & 3 & \begin{tabular}{|l|l|}
4 & 2 \\
\end{tabular} \\
\hline & RefazerProvasPenalidade[0] & 1 & 1 & 2 & 1 & 2 & 1 & 1 & & 1 & 2 & 1 & $\overline{4}$ & 1 & \begin{tabular}{l|l|}
1 & 2 \\
\end{tabular} \\
\hline & RefazerProvasPenalidade[50] & 1 & 4 & 1 & & 2 & & 1 & & 1 & 22 & 5 & & 4 & \begin{tabular}{|l|l|}
1 & 2 \\
\end{tabular} \\
\hline & NovasProvas & 3 & & 22 & Is & 3 & 5 & 1 & & 3 & 3 & 4 & & 4 & \begin{tabular}{|l|l|}
5 & 4 \\
\end{tabular} \\
\hline \multirow{6}{*}{$\begin{array}{c}\text { IF provasRespondidas }<=19 \text { AND } \\
\text { exerciciosCorretos }<=252 \text { AND } \\
\text { atividadesRealizadas }<=2\end{array}$} & AtividadesNaoRespondidasPenalidade $[0]$ & 5 & & 11 & & 5 & & 1 & & & 3 & $\sqrt{2}$ & & 1 & \begin{tabular}{|l|l|}
1 & 2 \\
\end{tabular} \\
\hline & AtividadesNaoRespondidasPenalidade[50] & 1 & & 22 & 2 & 1 & 1 & 5 & & 5 & 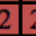 & $\overline{3}$ & & 4 & \begin{tabular}{|l|l|}
4 & 2 \\
\end{tabular} \\
\hline & AtividadeNivel $[\mathrm{X}]$ & 1 & 1 & 12 & $T 1$ & 3 & 1 & 4 & & 2 & 4 & 1 & $\overline{4}$ & 2 & \begin{tabular}{|l|l|l}
3 & 1 \\
\end{tabular} \\
\hline & RefazerAtividadesPenalidade $[0]$ & 1 & 2 & $3[2-200$ & 3 & 1 & 3 & 1 & & 1 & 2 & 3 & 4 & 1 & \begin{tabular}{|l|l|}
1 & 2 \\
\end{tabular} \\
\hline & ReverVideosUnidades $[\mathrm{X}]$ & 3 & 5 & $5]$ & & 5 & 5 & 3 & & 2 & 43 & te & & 2 & \begin{tabular}{|l|l|}
1 & 4 \\
\end{tabular} \\
\hline & ConteudoExterno $[\mathrm{X}]$ & 3 & & 5 & to & 4 & 2 & 1 & & 3 & 42 & 2 & & 2 & \begin{tabular}{|l|l|}
2 & 2 \\
\end{tabular} \\
\hline \multirow{6}{*}{$\begin{array}{c}\text { IF provasRespondidas }<=19 \text { AND } \\
\text { exercicios Corretos }<=252\end{array}$} & RefazerExerciciosPenalidade[0] & 1 & & 4 & & 1 & & 1 & & 1 & $45:$ & 4 & & 2 & \begin{tabular}{|l|l|}
1 & 2 \\
\end{tabular} \\
\hline & ReverVideosUnidades $[\mathrm{X}]$ & 3 & & 3 & & 5 & & 4 & & 4 & 3 ? & $\overline{4}$ & & 3 & \begin{tabular}{|l|l|}
1 & 4 \\
\end{tabular} \\
\hline & ConteudoExterno $[\mathrm{X}]$ & 4 & 4 & 4 & 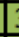 & 4 & 2 & 2 & & 5 & 3 & 3 & & 3 & \begin{tabular}{|l|l|}
4 & 3 \\
\end{tabular} \\
\hline & ExerciciosExtra & 5 & & 4 . & & 5 & 4 & 4 & & 4 & $45:$ & 5 & & 4 & \begin{tabular}{|l|l|}
5 & 4 \\
\end{tabular} \\
\hline & RefazerExerciciosNivel[facil] & 2 & & 21 & & 1 & 1 & 5 & & 2 & 3 : & 5 & & 2 & \begin{tabular}{|l|l|}
2 & 1 \\
\end{tabular} \\
\hline & RefazerExerciciosNivel[medio] & 2 & & 2 & & 1 & & & & 2 & 3[ & 3 & & 2 & \begin{tabular}{|l|l|l}
2 & 1 \\
\end{tabular} \\
\hline \multirow{5}{*}{$\begin{array}{c}\text { IF interacoesForum }<=3 \text { AND } \\
\text { interacoesChat }=0\end{array}$} & SolicitarAcessoChat & 4 & & 1 & & 5 & & 5 & & 3 & & 5 & & 4 & \begin{tabular}{|l|l|}
4 & 3 \\
\end{tabular} \\
\hline & AtividadeChat & 4 & & & & 4 & & & & 4 & 3 & 5 & & 1 & \begin{tabular}{|l|l|}
4 & 3 \\
\end{tabular} \\
\hline & InteracaoChat & 5 & & 5 & & 5 & & 3 & & 4 & 3 & Is & & 2 & \begin{tabular}{|l|l|}
3 & 4 \\
\end{tabular} \\
\hline & AnunciarAtividadesChat & 3 & & & & 5 & & 5 & & & & Ts & & 4 & \begin{tabular}{|l|l|}
3 & 5 \\
\end{tabular} \\
\hline & AtividadeExtraChat & 4 & & & & 5 & 1 & 4 & & 3 & 7 & & & 4 & \begin{tabular}{|l|l|l|}
3 & 4 \\
\end{tabular} \\
\hline
\end{tabular}

\section{CONCLUSÃO}

Nessa dissertação objetivou-se a criação, e validação (através de um estudo de caso), de uma ferramenta capaz de auxiliar professores de cursos baseados na web, a extrair informações relevantes das interações desses alunos com o ambiente de aprendizagem, através de tácnicas de Mineração de Dados Educacionais. Esses dados foram utilizados para realizar recomendações pedagógicas que promovessem o aprimoramento da experiência de aprendizado dos alunos.

Para atingir esse objetivo utilizamos o Processo de Recomendação Pedagógica, que orienta a descoberta de informações úteis, através da sinergia da Inteligência Humana e da Inteligência Artificial, tendo como finalidade prover apoio à tomada de decisões pedagógicas, para promover o aprimoramento da experiência de aprendizado para alunos e turmas [PAIVA, 2012].

Realizamos, por fim, um estudo de caso com dados de um ambiente de aprendizagem real, para avaliar a aplicabilidade da ferramenta. Através de tal estudo constatamos que a ferramenta é aplicável e traz benefícios ao processo de ensino e aprendizagem, uma vez que auxilia professores não apenas detectando práticas, mas, também, descobrindo os padrões que justificam tais práticas e oferecendo recomendações pedagógicas para as situações identificadas.

\section{REFERÊNCIAS}

BAKER, R.S.J.d. Data Mining for Education. McGaw, B., Peterson, P., Baker, E. (Eds.) International Encyclopedia of Education. Elsevier, 2010, ed. 03, vol. 07, pp. 112-118.

BAYER, Jaroslav; BUDZOVSKA, Hana; GERYK, Jan; OBSIVAC, Tomás; POPELINSKY, Lubomir. Predicting drop-out from social behaviour of students. Educational Data Mining Conference, 2012.

BITTENCOURT, Ig Ibert ; COSTA, Evandro de Barros ; Marlos Silva ; SOARES, Elvys . A Computational Model for Developing Semantic Web-based Educational Systems. Knowledge-Based Systems, 2009, vol. 22, pp. 302-315. 
II Congresso Brasileiro de Informática na Educação (CBIE 2013)

Workshops (WCBIE 2013)

BITTENCOURT, Ig Ibert ; COSTA, Evandro de Barros. Modelos e Ferramentas para a Construção de Sistemas Educacionais Adaptativos e Semânticos. Revista Brasileira de Informação na Educação, 2011, vol. 19, pp. 85-98.

BOUCKAERT, Remco R.; FRANK, Eibe; HALL, Mark; KIRKBY, Richard; REUTEMANN, Peter; SEEWALD, Alex; SCUSE, David. WEKA Manual for Version 3.6.8. University of Waikato, 2012.

BRITO, Patrick H. S.; BITTENCOURT, Ig Ibert; COSTA, Evandro; HOLANDA, Olavo; FERREIRA, Rafael; RIBEIRO, Thiago. A Systematic Approach for Designing Educational Recommender Systems. Educational Recommender Systems and Technologies, IGI Global, 2012.

DE CARVALHO, André C. Ponce de Leon F.; BRAYNER, Angelo; Et.Al. Grandes Desafios da Pesquisa em Computação no Brasil 2006 - 2016. Disponível em http://www.gta.ufrj.br/rebu/arquivos/SBC-Grandes.pdf. Acessado em 12/11/2012.

DESAFIE!. I Workshop de Desafios da Computação Aplicada à Educação. Disponível em: http://www.imago.ufpr.br/csbc2012/desafie.php. Acessado em: 14/05/2013.

DUKE. Bioelectricity: A Quantitative Approach. Duke University's First MOOC. Disponível em: http://dukespace.lib.duke.edu/dspace/handle/10161/6216. Acessado em: 27/03/2013. Fevereiro, 2013.

GAYARD, Leonel Aguilar. RUBIRA, Celília M. F. GUERRA, Paulo A. de Castro. COSMOS: A Component System Model for Software Architectures. Universidade Estadual de Campinas. Instituto de Computação, 2008.

GIBERT, Karina; IZQUIERDO, Joaquín; HOLMES, Geoff; ATHANASIADIS, Ioannis; COMAS, Joaquim; SÀNCHEZ-MARRÈ, Miquel. On the role of pre and post-processing in environmental data mining. International Congress on Environmental Modeling and Software, 2008.

HAN, Jiawei; KAMBER, Micheline. Data Mining: Concepts and Techniques, 2011, ed. 03 - Elsevier/Morgan Kaufmann.

HUANG, Shaobo; FANG, Ning. A Work in Progress: Early Prediction of Students' Academic Performance in an Introductory Engineering Course Through Different Mathematical Modeling Techniques. Frontiers in Education, 2012.

KANELLOPOULOS, Dimitris; KOTSIANTIS, Sotiris. Towards an ontology-based system for intelligent prediction of student dropouts in distance education. International Journal of Management in Education. Vol 2, No. 2, 2008.

MORAN, José Manoel. O que aprendi sobre avaliação em cursos semi-presenciais. In: SILVA, Marco; SANTOS, Edméa (Orgs). Avaliação da Aprendizagem em Educação Online. São Paulo: Loyola, 2006. Disponível em: http://www.eca.usp.br/prof/moran/aprendi.html. Acessado em: 03/07/2013.

PAIVA, Ranilson O. Araújo; BITTENCOURT, Ig Ibert; PACHECO, Henrique; SILVA, Alan Pedro da; JACQUES, Patrícia; ISOTANI, Seiji. Mineração de Dados e a Gestão Inteligente da Aprendizagem: Desafios e Direcionamentos in XXXII Congresso da Sociedade Brasileira de Computação, 2012.

PARK, Ji-Hye; CHOI, Hee Jun. Factors Influencing Adult Learners'Decision to Drop Out or Persist in Online Learning. Educational Technology \& Society, 2009, vol. 12, pp 207-217.

PARK, Ji-Hye. Factors Related to Learner Dropout in Online Learning, $2007 . \quad$ Disponível em: http://www.eric.ed.gov/ERICWebPortal/recordDetail?accno=ED504556. Acessado em: 03/07/2013.

PNE. Portal do Mec. Plano Nacional de Educação - PNE. Disponível em: http://portal.mec.gov.br/index.php?option=com_content\&view=article\&id=16478\&Itemid=1107. Acessado em: 24/04/2012.

PNE(b). Projeto de Lei 8035/2010. Câmara dos Deputados. Disponível em: http://www.camara.gov.br/proposicoesWeb/fichadetramitacao?idProposicao=490116. Acessado em: 12/04/2012.

RICCI, Francesco; ROKACH, Lior; SHAPIRA, Bracha; KANTOR, Paul B. Recommender Systems Handbook. Springer, 2011.

ROMERO, Cristóbal; VENTURA, Sebastian; PECHENIZKIY; BAKER, Ryan. Handbook of Educational Data Mining.Florida: CRC Press, 2011

SALMON, Gilly. E-Moderation - The Key to Teaching and Learning Online. Kogan Page, 2000.

SOMMERVILLE, Ian. Software Engineering. ed. 09. Addison-Wesley, 2011.

UFAL Línguas. Disponível em: http://www.ufal.edu.br/cied/informes/cied-torna-publico-o-edital-do-curso-201cel-mundo-hispanico-sinfronteiras. Acessado em: 15/01/2013.

WALKER, Maurice. PISA 2009 Plus Results: Performance of 15-year-olds in Reading, Mathematics and Science for 10 Additional Participants. Acer Press, 2011.

WITTEN, Ian; FRANK, Eibe; HALL, Mark. Data Mining: Practical Machine Learning Tools and Techniques. Elsevier, 2011. ed. 3. Massachusetts.

WU, Xindong; et. Al. Top 10 Algorithms in Data Mining. Knowledge Information Systems, 2007 - Springer. 